\title{
Factors Involved in Selection of a Career in Surgery and Orthopedics for Medical Students in Malawi
}

\section{C.Kollias ${ }^{1}$, L.Banza ${ }^{2}$, N.Mkandawire ${ }^{2}$}

1. Division of Orthopaedic Surgery, University of Calgary

2.Dept of Surgery, College of Medicine programme. One further non-Malawian is currently nearing the end of Orthopaedic training.

Several theories have been suggested informally by surgical personnel in Malawi regarding the cause for the apparent lack of interest in surgery and Orthopaedics amongst medical trainees. These have included factors such as fear of occupational HIV exposure, lifestyle, and lack of resources to treat patients. In Western countries, surgical career selection has been evaluated and lifestyle has been cited as a key deterrent from a surgical career. ${ }^{1-3}$ In the African context, there is very little known about medical trainee career selection. ${ }^{4-8}$ Furthermore, there is no published literature to date that has investigated surgical or Orthopaedic career selection amongst African medical trainees. Additionally, to date there has never been an evaluation of career selection amongst medical students in Malawi. With the goal of facilitating recruitment into Orthopaedic Surgery in Malawi, we undertook this study in an effort to evaluate the desirability of various surgical and non-surgical specialties. We also wanted to explore Malawian Medical Students' rationale in choosing a particular specialty, specifically Surgery and Orthopaedics.

\section{Materials and Methods}

An anonymous on-line internet survey was constructed denovo through a described structured process. ${ }^{9}$ This included Item Generation in the form of a literature review from the developing world as well as Western nations, informal focus groups with Malawian Medical Students, and interviews with the single Malawian Orthopaedic Surgeon as well as foreign Surgeons from other African nations and the United Kingdom. Following this, Item Reduction was performed to eliminate any redundant areas of questioning. The survey was then composed and formatted. We used a 35-item survey to collect demographic data and career preferences as well as a Likert scale format to examine student views and opinions. The survey was then pre-tested by several visiting British Medical Students and further input was given by the Malawian Orthopaedic Surgeon. An Assessment of Validity was then performed by a recognized survey content expert. Further modifications were made and the survey was Pilot-Tested in on-line format by several Malawian Medical Students. Minimal clarifications were required and we proceeded to formal distribution and survey testing.

All medical students in their clinical rotation years (3-5) were given a 4 week period to voluntarily participate in the survey. The survey was administered in English, which is the language of instruction at Malawi College of Medicine. All students had good access to the internet. We received clearance to proceed with the survey from the College of Medicine Research and Ethics Committee (COMREC) at University of Malawi. Data was analyzed using Pearson Chisquare testing. 


\section{Results}

Of the 147 students in clinical years 3-5 in the College of Medicine, 70 participated in the survey giving a response rate of $48 \%$. The average age of respondents was 22.3 years and females represented $17 \%$ of respondents. Females represented $28 \%$ of the actual population of potential respondents.

Thirty-six percent of respondents were third year students; fourth and fifth year students represented $42 \%$ and $22 \%$ respectively. The majority of students reported growing up in an urban area (77\%) with the rest reporting a rural origin. All female respondents were from urban areas. Surgery was the most popular specialty with $46 \%$ of students indicating it as their first choice area. The next most popular choices were Internal Medicine (16\%), Paediatrics(15\%), Pathology (6\%), Obstetrics \& Gynaecology (4\%), Radiology (4\%), General Practice $(1 \%)$, Anaesthesia $(0 \%)$ and Psychiatry $(0 \%)$. Seven percent of students were undecided regarding a first choice specialty. (Figure 1)

Results across years were quite similar with first choice area as surgery for $44 \%$ of third year students, $45 \%$ of fourth year students and $50 \%$ of final year students. One third of female respondents identified surgery as their first choice specialty. Amongst the surgical specialties, 27\% of students chose General Surgery as their first choice subspecialty, followed by Neurosurgery (22\%), Orthopaedics (19\%), Cardiothoracic Surgery (18\%), Ophthalmology (4\%), Plastic Surgery $(2 \%)$ and Ear Nose Throat Surgery $(2 \%)$. Seven percent of students disliked surgery (Figure 2).

Among respondents, only 28\% indicated that they had received career guidance in Medical School and 7\% of students stated their families were influencing them to enter a certain specialty. Twenty-eight percent of students would prefer to do their Registrar/Residency training outside of Malawi even if this meant choosing a less desirable specialty. Eighty-four percent of students felt that the ability to improve the quality of life for patients within a specialty was an important factor in their career choice. Lifestyle was identified as an important factor in specialty selection as $80 \%$ and $83 \%$ of students indicated this was an important consideration at both the training level and consultant level respectively. Only 16\% of students felt that Orthopaedic training would take too long to complete and $23 \%$ believed they would have to work too many hours as an Orthopaedic Surgeon.

Employment options and the availability of Consultant jobs were major concerns to $87 \%$ of students. The majority of respondents $(75 \%)$ thought they would have good employment options as an Orthopaedic Surgeon in Malawi. Overall, $70 \%$ of students cited financial remuneration as a key factor in their choice of specialty. Twenty-five percent of students believed Orthopaedic Surgeons are well paid compared to other specialties, whereas $68 \%$ of students were undecided on this issue.

Amongst students, there was a genuine concern of occupational exposure to HIV. For those trainees who selected Surgery as their first choice specialty, 67\% indicated they were worried about acquiring HIV through a scalpel or needle stick injury. However, $68 \%$ of non-Surgery oriented students also indicated this concern. As such, fear of acquiring HIV by occupational injury did not appear to be a deterrent from choosing Surgery as a specialty choice $(\mathrm{p}=0.9)$.
Mentorship was a substantial influence on specialty selection as the vast majority of students $(92 \%)$ stated that positive mentors are a key factor on their choice of career. However, only $10 \%$ of students were able to identify a Surgery mentor and $14 \%$ were able to identify a mentor in Orthopaedics. Students who could identify a mentor in Orthopaedics were significantly more likely to choose Orthopaedic Surgery as their first choice specialty $(\mathrm{p}=0.01)$.

Many students seemed to be underexposed to Orthopaedics from a knowledge base as well as career exposure standpoint, with only $33 \%$ of students indicating they had sufficient exposure to Orthopaedics to make an informed career decision. Seventy-one percent of students stated they would like more exposure to Orthopaedics as a Medical Student or Intern.

\section{Discussion}

Malawi has a critical shortage of Orthopaedic Surgeons with 0.06 per 100,000 people. This is in grave contrast to Western nations like Canada where there are 3.1 Orthopaedic Surgeons for every 100,000 people ${ }^{10}$ the United Kingdom $(6.6 \text { per } 100,000)^{11}$ and the United States $\left(7.6\right.$ per 100,000). ${ }^{12}$ With the view of facilitating recruitment into Orthopaedic Surgery in Malawi we undertook this study to understand the critical elements of medical trainees' choice of specialty and their rationale in choosing an Orthopaedic career.

While there have been many studies in the literature evaluating medical student specialty career selection in Western nations, there is very minimal literature exploring this area in an African context., ${ }^{4,5,13}$ Surgical career selection amongst trainees has been evaluated in the evaluated in the United States, Europe and Australia by several authors. ${ }^{1,}$ $143,15,16$ However, there is very little known on this topic in an African setting. In Nigeria, previous literature has indicated that $21-41 \%$ of Medical Students favor Surgery as a first choice specialty., 8 In South Africa, the crisis of unfilled Consultant posts in General Surgery and decline in application for Registrar positions led to various interviews of trainees and Consultants, of which the collective views have been reported. ${ }^{6,7}$ Factors such as lack of financial remuneration and unattractive lifestyle were cited as key elements deterring trainees from a General Surgery career in South Africa. ${ }^{6}$ This is the first study to date evaluating factors involved in surgical and Orthopaedic career selection amongst trainees in any African setting.

Our findings clearly indicate that Surgery is the most popular career choice amongst Malawian Medical Students, which is promising for the future, despite evidence in South Africa to indicate surgery is losing popularity. ${ }^{6}$ This is in contrast to Psychiatry, also a notably underserviced specialty in Africa, which no students indicated as their first choice specialty. We do recognize possible non-response bias in our survey with a response rate of $48 \%$ which may have resulted in an overestimation regarding the popularity of Surgery and surgical subspecialties. Our survey response rate is comparable to other surveys of Medical personnel in an African setting. ${ }^{5}$

Our findings indicate that mentorship is likely the most important element in choice of a career. We also found mentorship to be a statistically significant factor in students' choice of a career in Orthopaedic Surgery. This is consistent with findings in a Western context, where positive 
interactions with attending Surgeons is believed to favorably influence students towards careers in Surgery. ${ }^{17}$ In addition to mentorship, our findings in an African setting also identify the need for more exposure to Orthopaedic Surgery within Medical School since the majority of students indicated they were uninformed about many details of the Orthopaedic profession. Practically, this is difficult to implement since the clinical demands on the single Malawian Orthopaedic Surgeon and the few foreign Surgeons are overwhelming. Some students suggested a role for scheduled career talks by Consultants within the various surgical specialties to increase student exposure.

We expected to identify some cultural and social factors unique to a Sub-Saharan African setting that would contribute to specialty selection. Students from Rural and Urban origins were equally likely to choose Surgery, while family influence and expectations had virtually no role on students' specialty choice. While financial remuneration and employment options were important to students, these did not appear to be the main factors in choice of specialty. There was a strong desire on the part of students to improve the quality of life and implement effective treatments for their patients. Given an HIV prevalence of $11.9 \%$ in Malawi, ${ }^{18}$ we suspected that the risk of occupational HIV exposure might deter Medical Students from selecting a career in Surgery, however our results do not support this. Indeed, the majority of students were concerned about this but students picking non-surgical careers were not any more likely to indicate this fear.

In summary, we believe that the future is promising for increasing the number of surgeons and specifically Orthopaedic Surgeons in Malawi. There is evidence that the majority $(60 \%)$ of graduates from the College of Medicine in Malawi stay in the country. ${ }^{19}$ Thus we suggest that as time progresses and trainees are successfully recruited into Orthopaedics, the process of mentorship will gain momentum exponentially. Future directions need to focus on mentorship both formally and informally, as well as securing funding for positions in post-graduate training in surgery. Furthermore, more exposure to Orthopaedics in the undergraduate medical program in the College of Medicine has been requested by Medical Students. Since remuneration appears to also be an important factor in specialty selection, we must also ensure that Surgeons are compensated appropriately relative to non-surgical medical specialties. We suggest that these findings may be reflective of many countries in sub-Saharan Africa, recognizing that there are unique political, ethnic, economic and social influences that are involved in various regions of the continent. Our hope is that this study will be a contribution to improving the musculoskeletal health for the 906 million people currently living in Sub-Saharan Africa and the generations proceeding. ${ }^{20}$

\section{References}

1. Ek EW, Ek ET, Mackay SD Undergraduate experience of surgical teaching and its influence and its influence on career choice. ANZ J Surg 2005;75:713-8.

2. Marschall JG, Karimuddin AA Decline in popularity of general surgery as a career choice in North America: review of postgraduate residency training selection in Canada, 1996-2001. World J Surg 2003;27:249-52.

3. O’Herrin JK, Lewis BJ, Rikkers LF, Chen H Why do students choose careers in surgery? J Surg Res 2004;119:124-9.

4. Adeboye A, Ayanniyi AA, mola-Popoola DS, Owoeye JF The choice of ophthalmology as a career among Nigerian medical interns. Afr J Med Med Sci 2006;35:321-3.

5. Erinosho OA Career goals of medical students in Nigeria. $J$ Med Educ 1977;52:215-7.

6. Kahn D, Pillay S, Veller MG, Panieri E, Westcott MJ General surgery in crisis--factors that impact on a career in general surgery. S Afr J Surg 2006;44:108-12.

7. Kahn D, Pillay S, Veller MG, Panieri E, Westcott MJ General surgery in crisis--the critical shortage. S Afr J Surg 2006;44:88-92, 94.

8. Oyebola DD, Adewoye OE Preference of preclinical medical students for medical specialties and the basic medical sciences. Afr J Med Med Sci 1998;27:209-12.

9. Burns KE, Duffett M, Kho ME, Meade MO, Adhikari NK, Sinuff T, Cook DJ A guide for the design and conduct of self-administered surveys of clinicians. CMAJ 2008;179:801-2.

10. Comeau P Crisis in orthopedic care: surgeon and resource shortage. CMAJ 2004;171(3):223.

11. No authors listed. British Orthopaedic Association. http://www.boa. ac.uk/en/about-boa/what-we-do/(date last accessed 31 May 2009).

12. Iorio R, Robb WJ, Healy WL, Berry DJ, Kyle RF, Lewallen DG, Trousdale RT, Jiranek WJ, Stamos VP, Parsley BS Orthopaedic Surgeon Workforce and Volume Assessment for Total Hip and Knee Replacement in the United States: Preparing for an Epidemic. J Bone Joint Surg Am 2008;90:1598-605.

13. Jombo GT Pathology as a profession: contribution of undergraduate training on choice of career among final year students of a medical school in north central Nigeria. Niger J Med 2006;15:309-13.

14. Andriole DA, Klingensmith ME, Jeffe DB Who are our future surgeons? Characteristics of medical school graduates planning surgical careers: analysis of the 1997 to 2004 Association of American Medical Colleges' Graduation Questionnaire National Database. J Am Coll Surg 2006;203:177-85.

15. Cochran A, Paukert JL, Neumayer LA Does a general surgery clerkship influence student perceptions of surgeons and surgical careers? Surgery 2003;134:153-7.

16. Sherry E, Mobbs R, Henderson A Becoming an orthopaedic surgeon: background of trainees and their opinions of selection criteria for orthopaedic training. Aust N Z J Surg 1996;66:473-7.

17.Kozar RA, Lucci A, Miller CC, Azizzadeh A, Cocanour CS, Potts JR, Fischer CP, Brundage SI Brief intervention by surgeons can influence students toward a career in surgery. J Surg Res 2003;111:166-9.

18. No authors listed. Unicef - Info by Country (Malawi). http://www. unicef.org/infobycountry/malawi.html(date last accessed 31 May 2009).

19. Zijlstra EE, Broadhead RL The College of Medicine in the Republic of Malawi: towards sustainable staff development. Hum Resour Health 2007;5:10-5.

20. No authors listed. UNFPA Worldwide Sub-Saharan Africa. http:// www.unfpa.org/worldwide/africa.html(date last accessed 31 May 2009). 


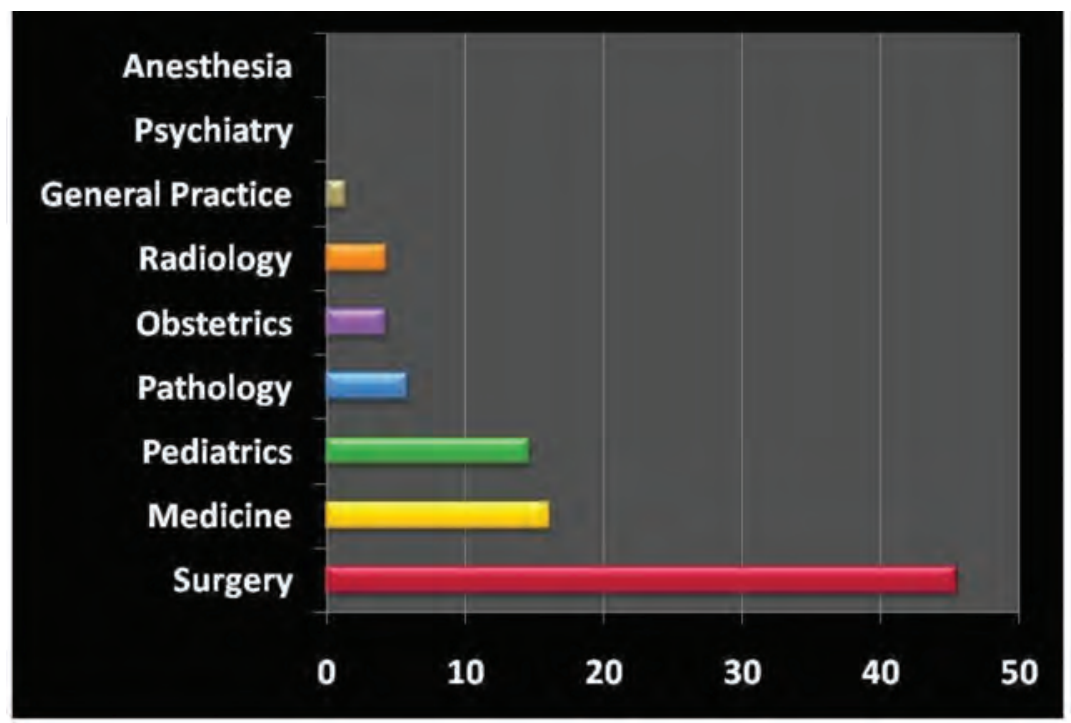

Figure 1: Medical Students' most preferred specialty by percentage of respondents. $158 \times 107 \mathrm{~mm}(150 \times 150 \mathrm{DPI})$

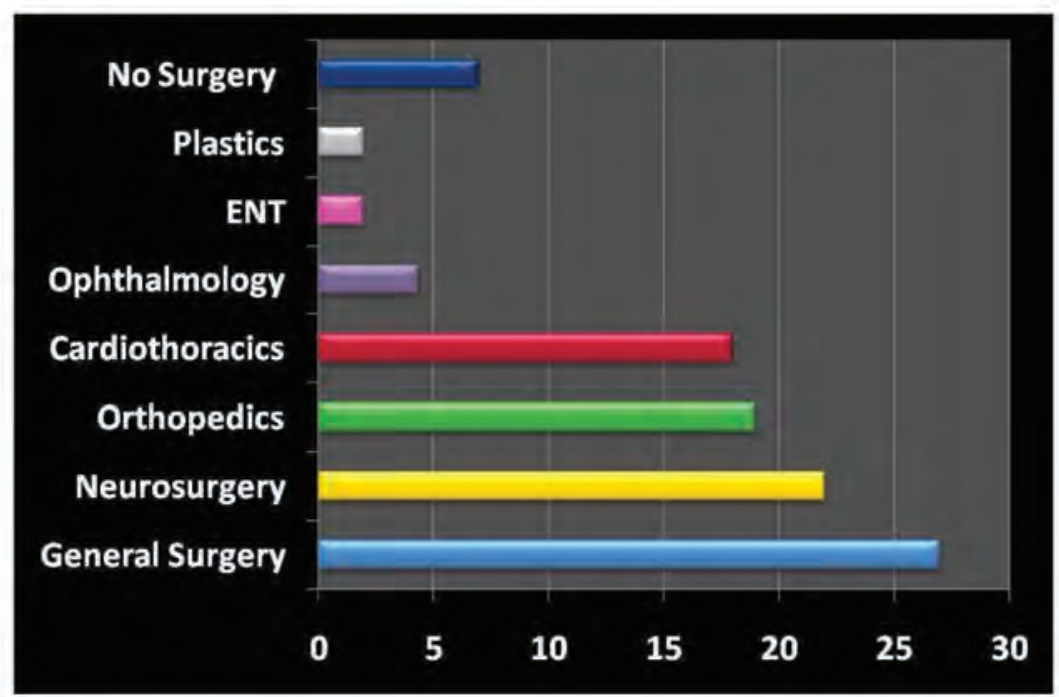

Figure 2: Medical Students' most preferred surgical specialty by percentage of respondents. $159 \times 104 \mathrm{~mm}(150 \times 150 \mathrm{DPI})$ 